\title{
Tunable optofluidic microlasers based on optically stretched emulsion droplets
}

\author{
Mehdi Aas ${ }^{a}$, Alexandr Jonáš ${ }^{a}$, Alper Kiraz ${ }^{a, c}$, Oto Brzobohatý $^{b}$, Jan Ježek ${ }^{b}$, Zdeněk Pilát ${ }^{b}$, \\ and Pavel Zemánek ${ }^{b}$ \\ ${ }^{a}$ Department of Physics, Koç University, Rumelifeneri Yolu, Sariyer, 34450 Istanbul, Turkey; \\ ${ }^{b}$ Institute of Scientific Instruments of the ASCR, v.v.i., Královopolská 147, 61264 Brno, Czech \\ Republic; \\ ${ }^{c}$ Koç University TÜPRAŞ Energy Center (KUTEM), Koç University, Rumelifeneri Yolu, \\ Sariyer, 34450 Istanbul, Turkey
}

\begin{abstract}
We introduce tunable optofluidic microlasers based on optically stretched, dye-doped emulsion droplets confined in a dual-beam optical trap. Optically trapped microdroplets of oil emulsified in water and stained with fluorescent dye act as active ultrahigh-Q optical resonant cavities hosting whispering gallery modes (WGMs) which enable dye lasing with low threshold pump powers. In order to achieve tunable dye lasing, the droplets are pumped with a pulsed green laser beam and simultaneously stretched by light in the dual-beam trap. For a given stretching power, the magnitude of the droplet deformation is dictated by the interfacial tension between the droplet and the host liquid which is adjustable by adding surfactants. Increase of power of the dual-beam trap causes a directly proportional change of the droplet stretching deformation. Subsequently, resonant path lengths of different WGMs propagating in the droplet are modified, leading to shifts in the corresponding microlaser emission wavelenghts. Using this technique, we present all-optical, almost reversible spectral tuning of the lasing WGMs and show that the direction of wavelength tuning depends on the position of the pump beam focus on the droplet, consistent with the deformation of originally spherical droplet towards a prolate spheroid. In addition, we study the effects of changes of the droplet and immersion medium temperature on the spectral position of lasing WGMs and demonstrate that droplet heating leads to red-tuning of the droplet lasing wavelength.
\end{abstract}

Keywords: Optical Stretcher, Microdroplet, Tunable Laser, Whispering Gallery Mode, Dye Lasing, Nonlinear Optics, Optical Trapping

\section{INTRODUCTION}

Liquid droplets have been long recognized and studied for their peculiar optical properties. ${ }^{1}$ At the microscale, shape of liquids is dictated by the liquid surface tension that tends to minimize liquid surface area, thus transforming a small liquid parcel into a perfect sphere. Such spherical droplets with exceptionally smooth surfaces can act as optical cavities hosting morphology-dependent resonances called whispering gallery modes (WGMs) that are confined to small volumes near the droplet rim. ${ }^{2}$ Consequently, very high electric field intensities can be achieved in microdroplets that can mediate strong coupling between light and quantum systems such as atoms, molecules, or quantum dots. ${ }^{3}$

Droplet-based optical resonators have found a number of applications in optofluidics - an exciting new research field that exploits unique properties of fluids for creating optical components and systems. ${ }^{4}$ Dye lasing, ${ }^{5}$ stimulated Raman scattering, ${ }^{6}$ and Raman lasing ${ }^{7}$ in microdroplets have been demonstrated at low threshold pump powers. Apart from their significance for the fundamental physics research, microdroplets are well suited to another important line of applications that benefit from the sensitivity of the WGMs to the microdroplet size

Further author information: (Send correspondence to A.J., A.K. and P.Z.)

A.J.: E-mail: ajonas@ku.edu.tr, Telephone: +90212338 1588

A.K.: E-mail: akiraz@ku.edu.tr, Telephone: +90212338 1701

P.Z.: E-mail: pavlik@isibrno.cz, Telephone: +420 541514202

Optical Trapping and Optical Micromanipulation X, edited by Kishan Dholakia, Gabriel C. Spalding, Proc. of SPIE Vol. 8810, 88101Y · (c) 2013 SPIE · CCC code: 0277-786X/13/\$18 · doi: 10.1117/12.2024252 
as well as the refractive indices of the droplet liquid and the surrounding medium. These properties naturally make microdroplets very attractive in biological and chemical sensing, and in developing optical devices that necessitate a tunable optical microcavity, e.g. tunable optical switches or light sources. ${ }^{8}$

Tunability of shape, size, and composition is an important advantage of reconfigurable optofluidic components based on microdroplets. The most straightforward way of tuning spectral position of WGMs of airborne droplets relies on controlled changes of the liquid cavity size using evaporation/condensation, ${ }^{8}$ electrowetting, ${ }^{9}$ or photothermal effect. ${ }^{10}$ Adjustment of resonant frequency of microdroplet optical cavity can also be achieved by controlled deformation of the microdroplet which lifts the degeneracy of WGMs with different azimuthal mode numbers. Frequency splitting of the azimuthal WGMs then depends on the extent of the microdroplet deformation. ${ }^{11,12}$ For microdroplets surrounded by a solid elastomer, tuning has been achieved by mechanical shape deformation ${ }^{13}$ or by changing refractive index of liquid crystal droplets in external electric field. ${ }^{14}$ However, for the case of microdroplets surrounded by another liquid in microfluidic channels, reversible spectral tuning has not been shown yet, despite different demonstrations of optical functionality of emulsion droplets including dye lasing. ${ }^{15,16}$ In order to address the issue of spectral tuning, researchers have demonstrated multicolor dye lasing in microfluidic channels by fast switching between consecutive droplets doped with different dye molecules. ${ }^{17}$ Recently, emission wavelength tuning by continuous decrease of the droplet size due to dissolving of benzyl alcohol microdroplets in water has also been demonstrated. ${ }^{18}$ Nevertheless, such dissolution-based tuning of the lasing emission wavelength is irreversible.

As shown by Guck et al., transparent dielectric microobjects suspended in a liquid can be deformed by the forces of light. ${ }^{19}$ At the object surface where the material properties (in particular, the dielectric constant) change abruptly, transfer of momentum from light to the object occurs. Such surface momentum transfer then leads to surface stresses that can cause deformation of the illuminated object, with the amount of deformation directly proportional to the incident light power. Optically induced deformation of soft microobjects (lipid vesicles, living cells) has been successfully demonstrated using a pair of counter-propagating laser beams in the so-called optical stretcher geometry. ${ }^{19}$ Optical stretcher uses the dual-beam optical trap configuration ${ }^{20-22}$ which consists of two precisely aligned, slightly divergent counter-propagating laser beams emitted, for example, from optical fibers. Because of the Gaussian transversal intensity profile of both beams, high refractive index objects (e.g. oil droplets suspended in water) are pulled toward the beams axis and, subsequently, move along this axis to the location where the radiation pressures of both beams just balance each other. At this position, the objects are stably confined and their stretching in the optical field of the two beams can be studied.

Optical stretcher represents a convenient, all-optical method for tuning the emission spectra of lasing microdroplets via controlled droplet deformation that leads to frequency-splitting of degenerate WGMs of originally spherical droplets. However, the magnitude of optical stretching forces is rather small in comparison with the typical interfacial tension forces acting between two immiscible liquids in emulsions. In order to achieve sufficient droplet deformations without using prohibitively high stretching laser powers, interfacial tension has to be lowered by several orders of magnitude. This can be accomplished by using various mixtures of surfactant molecules and inorganic salts for the emulsion preparation. Emulsions with ultra-low interfacial tension have been used by Møller et al..$^{23}$ and Ward et al. ${ }^{24}$ in the single beam and multiple beam optical tweezers, respectively, to observe large deformations of the emulsion droplets with moderate trapping powers. Despite the above demonstrations, optical stretcher geometry has not been employed yet for light-induced tuning of the emission spectra of lasing microdroplets.

In this paper, we introduce novel tunable optofluidic dye microlasers that use optical stretching forces for the adjustment of the laser emission wavelength. Our microlasers are based on droplets of immersion oil emulsified in water and confined in a dual-beam optical trap. Since the droplets are stained with a fluorescent dye, they function as active ultrahigh-Q optical resonant cavities that are optically pumped with an independent pulsed green laser. In order to reduce the stretching power required for microlaser tuning, we lower the interfacial tension between the droplets and aqueous host medium to $\sim 1 \mathrm{mN} / \mathrm{m}$ using a surfactant and sodium chloride. We demonstrate almost reversible spectral tuning of the lasing WGMs with tuning slopes as large as $\sim 0.5 \mathrm{~nm} / \mathrm{W}$ (nm per Watt of the total stretching power). We also show that the direction of wavelength tuning depends on the position of the pump beam focus on the droplet. In addition, we characterize the effects of changes of the droplet and immersion medium temperature on the spectral position of lasing WGMs and show that these 
thermally induced spectral shifts are caused by simultaneous changes of the droplet size and refractive index of both the droplet and the host medium resulting from the droplet heating by the stretching laser beam.

\section{EXPERIMENTAL SETUP FOR MICRODROPLET LASING AND STRETCHING}

Experimental setup for the observation of tunable lasing from optically trapped and stretched emulsion microdroplets is shown in Fig. 1. Studied oil-in-water emulsions were contained within home-made PDMS microfluidic chips featuring a single channel with square cross-section of $160 \times 160 \mu \mathrm{m}^{2}$. These chips supported two multimode optical fibers with $50 \mu \mathrm{m}$ core and $125 \mu \mathrm{m}$ cladding diameters. The faces of the fibers were parallel to the opposite side walls of the channel and their optical axes were precisely aligned to form the dual-beam trap (see inset in Fig. 1a). The microfluidic channel had one inlet and one outlet with a syringe connected to the inlet via tubing to inject previously prepared microdroplet emulsions and washing solutions. Detailed manufacturing process for the chips is given in the next subsection. In order to observe the droplets, excite and collect the fluorescence emission from the droplets, and implement single beam optical trap, PDMS chips were mounted on an xyz stage of an inverted optical microscope equipped with a water immersion objective $(\mathrm{NA}=1.2,60 \mathrm{x}$; Nikon).

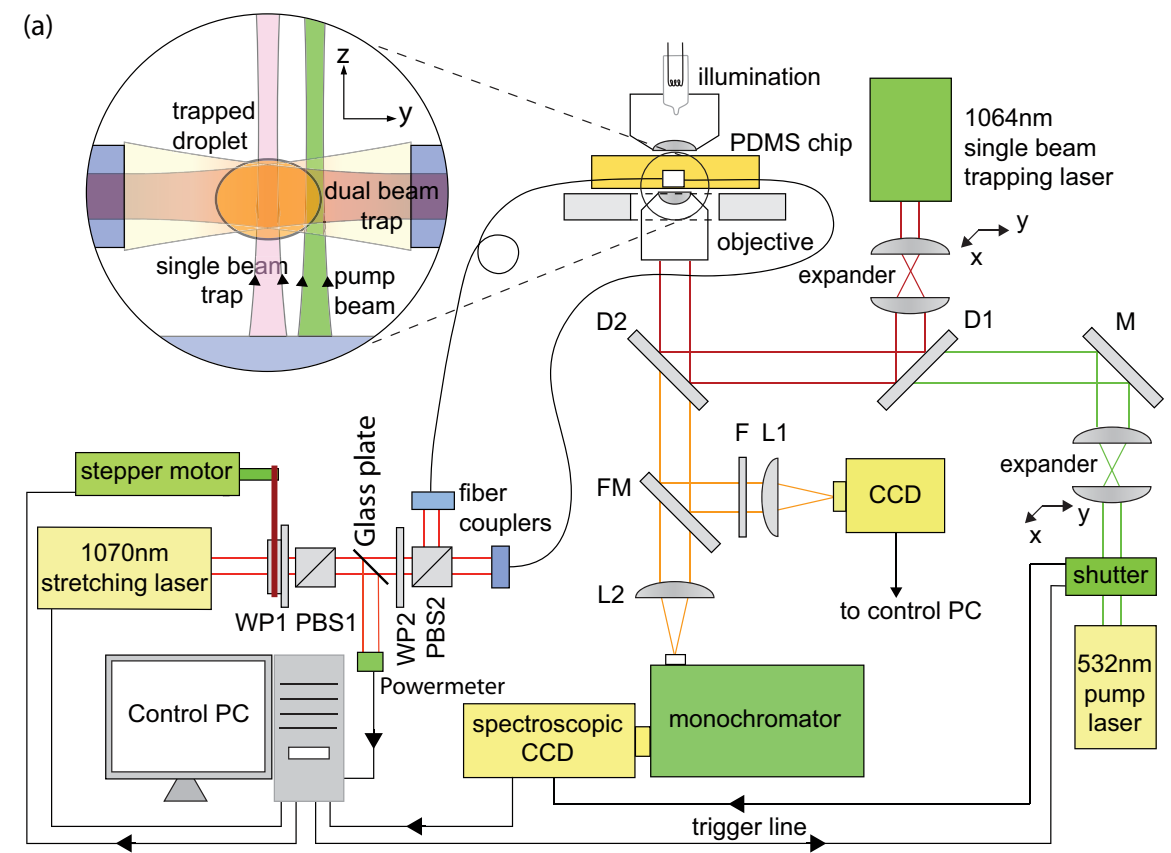

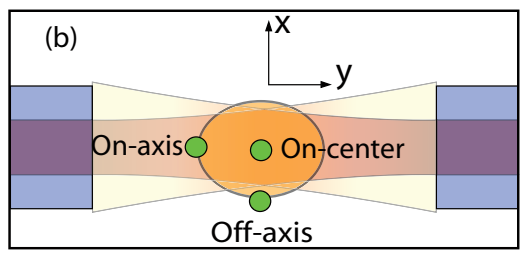

Droplet excitation geometry

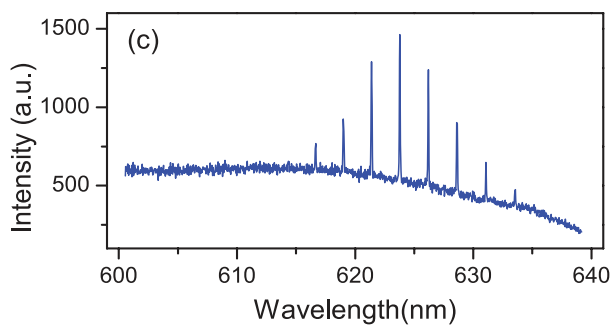

Figure 1. (a) Experimental setup schematics. The inset shows the geometry of the single- and dual-beam optical traps and the pump beam. (b) Droplet excitation geometry - view along z-axis, in the propagation direction of the pump beam. (c) A sample lasing spectrum of a $49 \mu \mathrm{m}$ diameter droplet with stretching power of $100 \mathrm{~mW}$.

Laser light source for the dual-beam trapping and optical stretching was a $1070 \mathrm{~nm}$ Ytterbium fiber laser (YLM-10-LP-SC; IPG Photonics) with $10 \mathrm{~W}$ maximum output power. Its output beam was directed to a $\lambda / 2$ 
waveplate (WP1) mounted on a stepper-motor-driven rotary stage and a polarizing beam splitter (PBS1) which together served to adjust the total stretching power coupled into the fibers. Following PBS1, another polarizing beam splitter PBS2 was used to divide the beam into two parts, with $\lambda / 2$ waveplate WP2 mounted on a manual rotary stage adjusting the division ratio of the total beam power between the fibers. Subsequently, both beams were coupled to respective single-mode patch cables (P5-980A-PCAPC-1; Thorlabs) using compact fiber couplers (PAF-X-11-C; Thorlabs). The output ports of the patch cables were then connected to the free ends of two multimode fibers embedded in the microfluidic chip. In order to measure the total actual power coupled to the fibers, a glass plate with an angle of 45 degrees relative to the laser beam was inserted in the beam path after PBS1; this plate reflected a small fraction of the total power $(\sim 5 \%)$ towards a power meter. Thus measured power fraction was calibrated by measuring the total output power from the bare fiber ends before inserting them into the chip.

Even though the power balance between the two stretching beams could be adjusted by WP2 for a constant total injected laser power, the power ratio displayed small fluctuations when the total injected power was modified by WP1. Consequently, a droplet confined in the dual-beam optical trap moved in all three directions with respect to the observation focal point when the total stretching power was altered. This phenomenon was further augmented by slight imperfections in the coaxial alignment of the two fibers. In addition, immersion oil used in the experiments was slightly denser than water and, thus, the oil droplets tended to sediment on the bottom of the microchannel. Since the transversal gradient optical force of the dual beam trap that counteracted gravity in our experimental arrangement (gravity pointing in negative $z$-direction) depends linearly on the total beam power, changes of the dual beam trapping power led to additional vertical displacement of the trapped droplet from the observation focal plane. Such unwanted droplet displacements changed the efficiency of the droplet pumping by the pulsed laser beam (see below) and, subsequently, the intensity of lasing emission from the trapped droplet. To overcome these issues and fix the position of the droplet in the axial and transversal directions during the droplet stretching, an additional infrared laser beam from a cw solid state laser (wavelength $1064 \mathrm{~nm}$, maximum power $300 \mathrm{~mW}$; CrystaLaser) was introduced to create an independent single beam trap (optical tweezers) perpendicular to the dual beam trap (see inset in Fig. 1a). This laser beam was sent through a 3x beam expander and reflected from two dichroic mirrors D1, D2 before being focused into the sample chamber by the water immersion microscope objective. The overall transmission of all optical elements in the single beam trapping path was measured to be about $20 \%$, resulting in the average trapping power of $\sim 60 \mathrm{~mW}$ at the specimen. Using the single beam trap, the studied droplet could be brought exactly to the position of the dual beam trap by adjusting the xyz stage of the inverted microscope. Furthermore, in order to change the position of the single beam trap in the objective focal plane $x y$, the first lens of the beam expander was mounted on an $x y$ stage that could be steered in $x$ and $y$ directions (perpendicular to the beam axis).

A $532 \mathrm{~nm}$ pulsed laser beam generated by frequency-doubling the output of a home-built passively Q-switched $\mathrm{Nd}-\mathrm{YVO}_{4}$ laser (20 ns pulse width, $33 \mathrm{kHz}$ repetition rate, maximal average output power $20 \mathrm{~mW}$ ) was used as the pump light source to excite fluorescently-stained trapped droplets. The pump laser beam was focused by the same microscope objective as the optical tweezers laser beam, after being transmitted through the dichroic mirror D1 and reflected from the dichroic mirror D2. The diameter of the pump beam focal spot was estimated from the beam spot images acquired after the beam reflection from the cover glass to be $1.24 \mu \mathrm{m}$. A $2 \mathrm{x}$ beam expander with the first lens mounted on an $x y$ stage was added to the optical path of the pump beam to steer the beam focus position in 3-D ( $x y$ stage was used to steer the focus of the beam in $x y$ direction and the distance between the two lenses of the beam expander was used to adjust its focal point position relative to the objective lens in the axial direction). This way, focusing points of the optical tweezers beam and the pump beam could be independently adjusted relative to each other and also relative to the imaging focal plane of the objective. Before the experiments, the droplet position was adjusted by moving the optical tweezers in the focal plane so that the focal spot of the pump laser lied near the droplet's rim for the maximal efficiency of the WGM excitation.

Fluorescence from a trapped microdroplet was collected using the same microscope objective as used for focusing the optical tweezers and pump beams, and dispersed by a monochromator (focal length $500 \mathrm{~mm}$; Acton Research) on the chip of a cooled CCD detector (Pixis 100; Princeton Instruments) controlled by WinSpec application software (Princeton Instruments). In Fig. 1c, a typical fluorescence spectrum of a trapped microdroplet is shown. The high-intensity WGMs located in the spectral interval 620 - $630 \mathrm{~nm}$ indicate droplet lasing. In 
order to prevent fast photobleaching of dye molecules inside the droplets, a shutter was added to the pump laser beam path; this shutter was only opened during the spectrum acquisition time and provided trigger signal for the spectroscopic detector to synchronize the data recording. To observe and take an image of the sample, the light in the imaging path of the microscope could be diverted to an ordinary CCD camera via a flippable mirror $(\mathrm{FM})$.

In order to automate the change of the stretching power and synchronize it with the collection of the droplet fluorescence spectra at each level of the stretching power, a LabVIEW code was developed to rotate the stepper motor, open the shutter for $8 \mathrm{~ms}$, and record the stretching laser power while, at the same time, externally triggered WinSpec software was collecting the droplet emission spectra with an exposure time of 8 ms. This way, the droplet deformation cycle could be carried out as fast as possible to minimize the effects of the droplet heating during the spectrum recording interval.

\section{MICROFLUIDIC CHIP FABRICATION}

Microfluidic chips for fiber-based dual-beam optical trapping and stretching were manufactured from PDMS elastomer by casting liquid PDMS precursor into a home-made reusable mold (master). The chip master was prepared in the following manner: first, a piece of optical fiber stripped of the plastic jacket with a diameter of $125 \mu \mathrm{m}$ was glued onto a cleaned glass substrate with a UV activated adhesive (Loctite 3922 or 3494). This fiber fragment served to form insertion ports for the dual-beam trap fibers in the chip. In the middle of the fiber length, two square capillary tubes (external width of $160 \mu \mathrm{m}$ ) were aligned coaxially with each other and perpendicularly to the fiber, one from each side of the fiber, and glued to the substrate. These capillary tubes defined the liquid channel in the chip. Central openings of the capillary tubes adjacent to the fiber were connected by UV activated adhesive. Resultant cross-like structure with a stalk formed by the capillary tubes and arms in the middle formed by the stripped optical fiber was placed into a plexiglass casting frame that was filled with liquid PDMS precursor and cured at the temperature of $\sim 85^{\circ} \mathrm{C}$ for 2 hours. After solidification, the resulting PDMS block was peeled off the mold and the PDMS surface was activated together with a $150 \mu \mathrm{m}$ thick microscope cover slip in an oxygen-argon microwave-excited plasma (oxygen-argon 1:1, total pressure $500 \mathrm{mTorr}$, microwave power $50 \mathrm{~W}$ ). Subsequently, the activated PDMS chip was pressed against the activated cover slip, thus sealing the chip permanently. The resulting chip featured one straight channel with square cross-section of $160 \times 160 \mu \mathrm{m}^{2}$ and two perfectly aligned cylindrical slots for optical fibers in the middle. The liquid inlet and outlet of the channel were realized during PDMS casting with a wire inserted into the mold or after PDMS solidification by a hole-punch.

In order to achieve optimal stretching forces for droplets with diameters around $50 \mu \mathrm{m}$, beam diameters at the droplet stable trapping position need to be comparable with the droplet size. This requirement was addressed by using multimode optical fibers (AFS50/125Y, NA=0.22; Thorlabs) with core diameters of $50 \mu \mathrm{m}$. These fibers were striped of their plastic jacket, cleaved, and inserted into the slots in the chip with the help of two small 3-D translational stages, one for each fiber. When the optimal positions of the fibers were reached, connections between the chip and the fibers were sealed using epoxy glue applied to the fiber slots from outside. In order to enable cleaning of the fibers, separation distance between the front faces of the fibers was chosen a little smaller than the width of microfluidic channel, equal to $134 \mu \mathrm{m}$.

\section{IMMERSION OIL/WATER EMULSION SYSTEM}

The emulsion system selected for our study consisted of microdroplets of dye-doped microscope immersion oil $\left(n=1.515, \rho=1.02 \mathrm{~g} / \mathrm{cm}^{3}\right.$, Merck) dispersed in deionized water $\left(n=1.334, \rho=1.00 \mathrm{~g} / \mathrm{cm}^{3}\right)$. This emulsion system of virtually immiscible liquids provides a high refractive index contrast together with a low density contrast between the droplet and the surrounding medium. This implies a good confinement of WGMs inside the trapped microdroplet, strong optical trapping force, and a small buoyancy that further facilitates the optical trapping of the droplets. The dye $\operatorname{DiI}(3)$ (1,1'-Dioctadecyl-3,3,3',3'-tetramethylindo carbocyanine perchlorate, Sigma Aldrich) that has a hydrophilic chromophore and hydrophobic side chains was selected as the laser gain medium. This dye has its excitation peak at $\sim 560 \mathrm{~nm}$ and emission peak at $\sim 575 \mathrm{~nm}$, and is well suited for the efficient excitation of the WGMs due to its surfactant nature that ensures its localization at the oil-water interface 
where WGMs also reside. ${ }^{16,25}$ In our experiments DiI(3) was dissolved in chloroform at $10 \mathrm{mM}$ concentration and the dye solution was subsequently added to the immersion oil in 2 to 100 ratio. This solution was put in the oven at $\sim 80^{\circ} \mathrm{C}$ and evacuated for $10 \mathrm{~min}$ to remove the chloroform content. Hence, the final solution contained only immersion oil with the dye concentration of $200 \mu \mathrm{M}$.

\section{CHARACTERIZATION AND ADJUSTMENT OF INTERFACIAL TENSION OF EMULSION DROPLETS}

Interfacial tension $(\gamma)$ between two immiscible liquids can be determined using Wilhelmy plate method.$^{26}$ In this method, contacting planar layers of studied liquids are formed in a container and a solid plate from a material that is wetted by the denser liquid is brought into contact with the liquid-liquid interface, perpendicularly to the interface. Subsequently, vertical force acting on the plate is measured that is directly proportional to $\gamma$. Since the denser phase in our experimental system was immersion oil, we used Wilhelmy plate made of Teflon that is wetted well by oil. Using Wilhelmy plate method, we measured the value of $\gamma \approx 13.1 \mathrm{mN} / \mathrm{m}$ for the dye-doped immersion oil/water interface.

Tuning of the lasing emission spectra of dye-doped microdroplets by optical stretching requires lowering of surface forces to the level comparable with optical forces. In order to achieve sufficiently low interfacial tensions, we added AOT surfactant (Docusate sodium salt; Sigma-Aldrich) and sodium chloride to the aqueous phase. This surfactant/salt system has been shown to decrease $\gamma$ to extremely low values (down to $0.001 \mathrm{mN} / \mathrm{m}$ ) depending on the concentration of both the surfactant and $\mathrm{NaCl}^{24,27}$ However, such low values of $\gamma$ are problematic in experiments where the droplets serve as optical resonant cavities since their shape can be easily disturbed by thermal fluctuations which cause degradation of the droplet cavity Q-factor. Here, we used $5 \mathrm{mM}$ AOT surfactant and $40 \mathrm{mM} \mathrm{NaCl}$ to decrease the interfacial tension to $\gamma=(1.5 \pm 0.1) \mathrm{mN} / \mathrm{m}$. We measured the interfacial tension in the presence of surfactant and salt in a range of temperatures between $25^{\circ} \mathrm{C}$ and $50^{\circ} \mathrm{C}$ and found a negligible variation of $\gamma$ with temperature. Thus, heating of droplets due to residual absorption of the stretching laser light does not cause any appreciable changes of surface forces.

Emulsion microdroplets were obtained by mixing the dye-doped immersion oil with the surfactant-salt-water solution in 1 to 50 ratio, and shaking the obtained mixture by hand. Droplets produced in this way had a large size distribution with diameters ranging from $<1 \mu \mathrm{m}$ up to $\sim 100 \mu \mathrm{m}$ and the emulsions were stable over $\sim 10$ hours. Prepared emulsions were subsequently loaded into the PDMS chip using liquid flow controlled by gravitational hydraulic pressure built by the height of water column in a $20 \mathrm{~cm}$ long piece of tubing connected to the inlet of the microchip. After loading, microdroplets were observed to float freely within the channel, thanks to their small buoyancy, without sticking to the walls of the sample chamber.

\section{OBSERVATION OF TUNABLE DYE LASING IN OPTICALLY STRETCHED MICRODROPLETS}

Experiments with tunable dye lasing in optically stretched microdroplets were carried out using multimode fibers in the configuration described in Section 3. In order to excite different WGMs in the stretched droplets, the focus of the pump beam was moved to different locations on the droplet.

First, the pump beam was focused at the rim of the droplet in the equatorial plane $x z$ perpendicular to the dual beam trap axis (off-axis excitation in Fig. 1b). Fig. 2a illustrates spectral tuning of three consecutive lasing WGMs with off-axis excitation for a droplet with the diameter of $47 \mu \mathrm{m}$ and $\gamma \approx 1.5 \mathrm{mN} / \mathrm{m}$. The total stretching power $P_{\text {stretch }}$ coupled into the two fibers was changed from $0 \mathrm{~W}$ to $0.88 \mathrm{~W}$ in two cycles and the total of 38 spectra were recorded with the minimal values of $P_{\text {stretch }}$ corresponding to frame numbers 1,19 , and 38 and the maximal values of $P_{\text {stretch }}$ corresponding to frame numbers 10 and 29 . Time interval between acquiring consecutive spectra was $50 \mathrm{~ms}$ and the droplet was exposed with the pump beam fluence of $\sim 490 \mathrm{~mJ} / \mathrm{cm}^{2}$ at the specimen for $8 \mathrm{~ms}$. From Fig. 2a, it is clear that the equatorial plane WGMs experience power-dependent blue tuning. As indicated by the black vertical line located near the middle WGM in Fig. 2a, the WGMs do not return back to their initial spectral positions after completing a single tuning cycle and a small blue drift of $\sim 0.04 \mathrm{~nm}$ is observed in all lasing WGMs. We interpreted this drift as a result of gradual dissolution of the droplet medium in water triggered by heating of the droplet by the stretching laser beams. This explanation was supported 
by the fact that the drift amount was directly proportional to the total duration of the tuning cycle and the maximal $P_{\text {stretch }}$ (data not shown). During data recording, the effect of the droplet dissolution was minimized by performing the experiments as fast as possible. In order to take the effect of residual droplet dissolution into consideration, we assumed a constant dissolution rate during each tuning cycle resulting in a linear drift of the WGM positions with time. Hence, WGM positions were corrected by subtracting a line connecting the first and last raw WGM position in the tuning cycle corresponding to $P_{\text {stretch }}=0 \mathrm{~W}$. This correction was applied to all of the tuning slope calculations presented in this paper. Figure $2 \mathrm{~b}$ shows the tuning of the lasing WGM wavelength for the central peak in Fig. 2a corrected for droplet dissolution as a function of $P_{\text {stretch }}$ for two subsequent tuning cycles. The slope of a linear fit to the experimental data is $-0.52 \mathrm{~nm} / \mathrm{W}$.
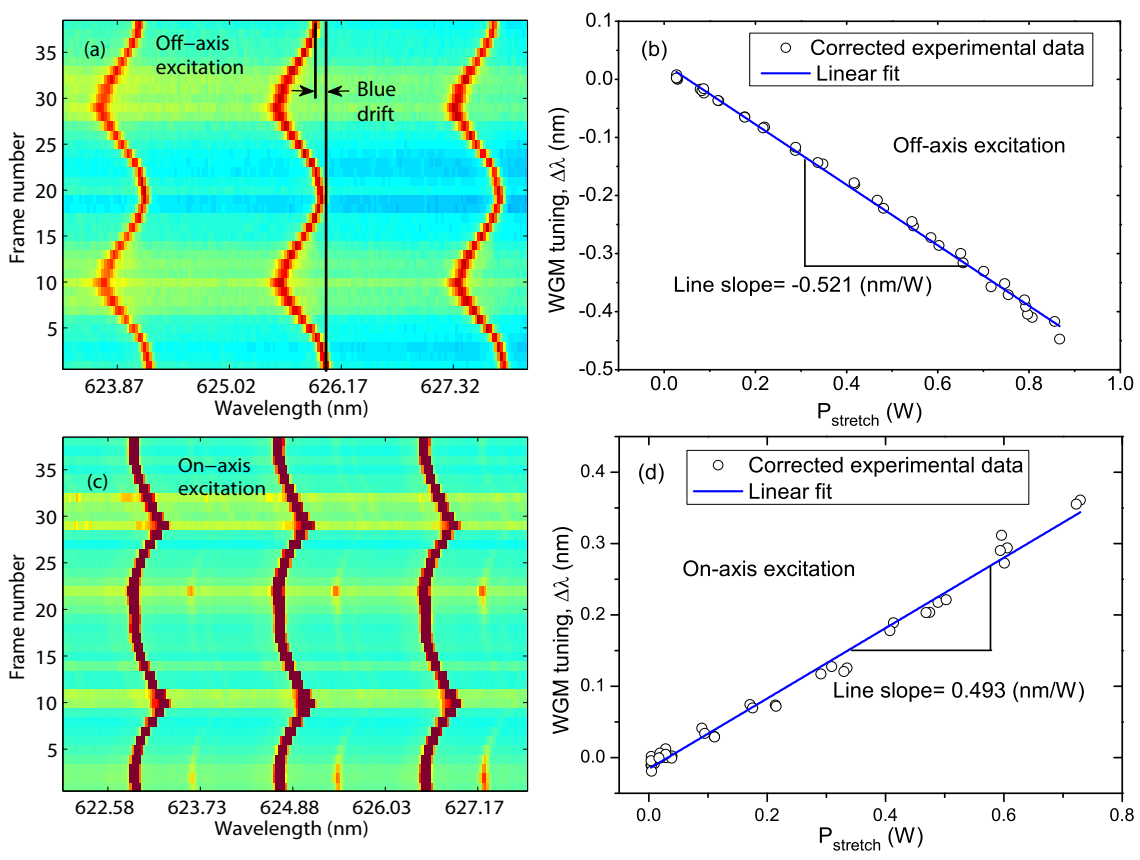

Figure 2. (a) Spectral tuning of lasing WGMs of a $47 \mu \mathrm{m}$ diameter droplet in off-axis excitation geometry (see Figure 1b) in the presence of surfactant (droplet interfacial tension $\gamma \approx 1.5 \mathrm{mN} / \mathrm{m}$ ). The black vertical line indicates blue drift caused by droplet dissolution. (b) Dissolution-corrected WGM tuning for the central peak in (a) as a function of the stretching laser power, $P_{\text {stretch. }}$ (c) Spectral tuning of lasing WGMs of a $49 \mu \mathrm{m}$ diameter droplet in on-axis excitation geometry (see Figure 1b) in the presence of surfactant (droplet interfacial tension $\gamma \approx 1.5 \mathrm{mN} / \mathrm{m}$ ). (d) Dissolution-corrected WGM tuning for the central peak in (c) as a function of the stretching laser power, $P_{\text {stretch }}$. Intensity values in arbitrary units increase from blue to red in (a) and (c).

In the second set of experiments, pump beam was focused at the droplet rim in the polar plane $y z$ containing the dual beam trap axis (on-axis excitation in Fig. 1b) and all other experimental parameters were kept fixed. The experiments in both configurations of the pump beam focus were carried out with different droplets since it was not possible to hold the droplet in the trap during the change of the pump beam position. Figure $2 \mathrm{c}$ shows spectral tuning of three consecutive lasing WGMs with on-axis excitation for a droplet with the diameter of $49 \mu \mathrm{m}$. $P_{\text {stretch }}$ was changed from $0 \mathrm{~W}$ to $0.75 \mathrm{~W}$ in two cycles and the total of 38 spectra were recorded with the minimal $P_{\text {stretch }}$ corresponding to frame numbers 1, 19, and 38 and the maximal $P_{\text {stretch }}$ corresponding to frame numbers 10 and 29. As in the previous experiment, time interval between acquiring consecutive spectra was $50 \mathrm{~ms}$ and the droplet was exposed with the pump beam fluence of $\sim 490 \mathrm{~mJ} / \mathrm{cm}^{2}$ at the specimen for 8 ms. As illustrated in Fig. 2d, for on-axis excitation, lasing WGMs experience red tuning that increases linearly with $P_{\text {stretch }}$. Here, a linear fit gives positive tuning slope of $0.49 \mathrm{~nm} / \mathrm{W}$. 

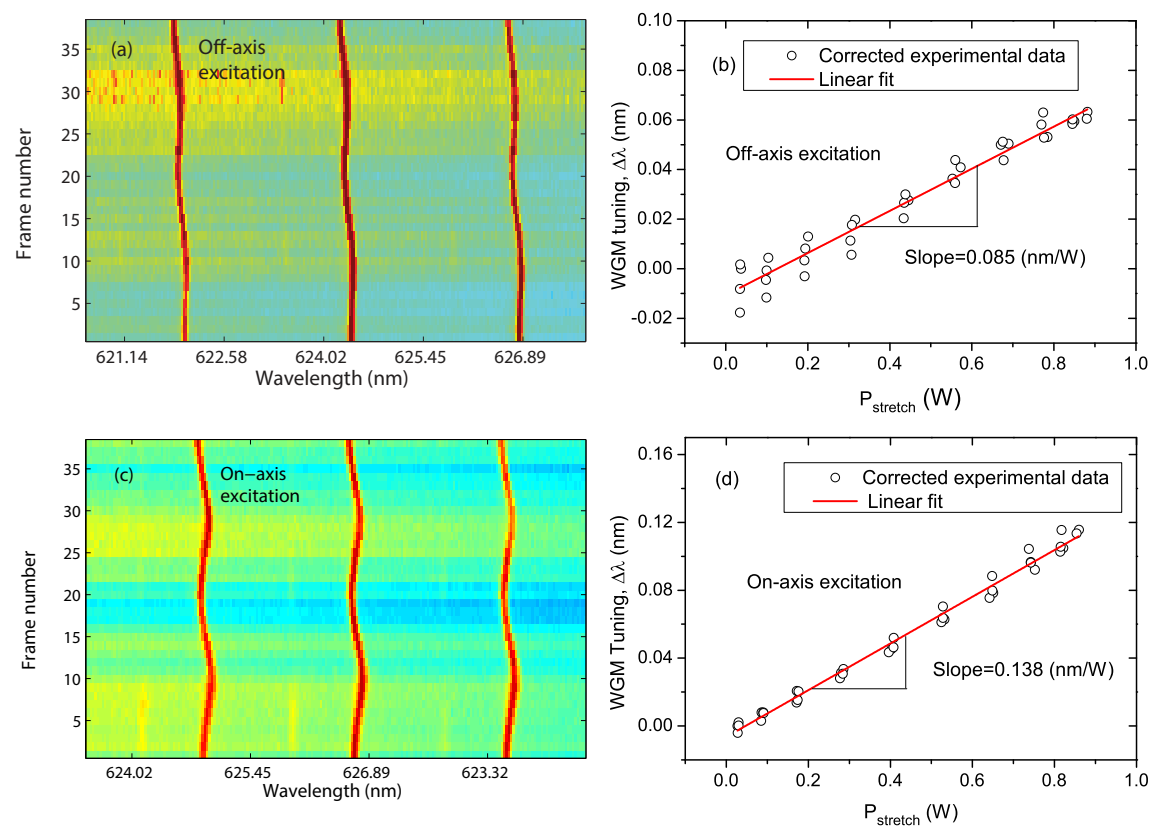

Figure 3. (a) Spectral tuning of lasing WGMs of a $44 \mu \mathrm{m}$ diameter droplet in off-axis excitation geometry (see Figure 1) without surfactant (droplet interfacial tension $\gamma \approx 13.1 \mathrm{mN} / \mathrm{m}$ ). (b) Dissolution-corrected WGM tuning for the central peak in (a) as a function of the stretching laser power, $P_{\text {stretch. }}$ (c) Spectral tuning of lasing WGMs of a $55 \mu$ m diameter droplet in on-axis excitation geometry (see Figure 1 ) without surfactant (droplet interfacial tension $\gamma \approx 13.1 \mathrm{mN} / \mathrm{m}$ ). (d) Dissolution-corrected WGM tuning for the central peak in (c) as a function of the stretching laser power, $P_{\text {stretch }}$. Intensity values in arbitrary units increase from blue to red in (a) and (c).

\section{INFLUENCE OF THERMAL EFFECTS ON WGM TUNING}

The asymptotic formula giving the spectral positions of WGMs with different azimuthal mode numbers as a function of the droplet ellipticity ${ }^{11,12}$ predicts the ratio of the spectral tuning slopes of the off-axis WGMs (azimuthal mode number $=$ angular momentum index) and the on-axis WGMs (azimuthal mode number $=0$ ) to be -2.0 for droplet sizes studied in this paper. However, in our experiments this ratio was observed to be -1.1 . In order to elucidate this difference, we carried out control experiments with dye-doped immersion oil droplets suspended in pure water without surfactant. As mentioned earlier, interfacial tension for oil/water system $\gamma \approx 13.1 \mathrm{mN} / \mathrm{m}$ is almost nine times larger than interfacial tension in the presence of surfactant $\gamma \approx 1.5 \mathrm{mN} / \mathrm{m}$. Consequently, one expects nine times smaller tuning slope for the surfactant-free emulsion system in comparison to the data presented in Fig. $2 \mathrm{~b}$ and Fig. 2d. ${ }^{23}$

In Fig. 3a, spectral tuning of lasing WGMs with off-axis excitation is shown for a $44 \mu \mathrm{m}$ diameter immersion oil droplet suspended in pure water. The excitation power, exposure time, and time between subsequent frames were identical to the experiments performed with droplets containing surfactant (Fig. 2). In the surfactant-free experiments, similarly to the previous cases with surfactant, a monotonous blue drift was observed resulting from the droplet dissolution. In addition, a small cyclic tuning of the WGM positions was also detected with changing $P_{\text {stretch }}$. However, unlike in the previous case with surfactant, spectral tuning for off-axis excitation was towards the red end of the spectrum. A line fit to the dissolution-corrected tuning data shown in Fig. 3b gives a positive tuning slope of $0.085 \mathrm{~nm} / \mathrm{W}$, in contrast to expected negative slope. In Fig. 3c, spectral tuning of lasing WGMs with on-axis excitation is shown for a $55 \mu \mathrm{m}$ diameter immersion oil droplet suspended in pure water. Again, a line fit to the dissolution-corrected tuning values in Fig. 3d shows a positive tuning slope of $0.138 \mathrm{~nm} / \mathrm{W}$ that has a proper sign but is larger than expected from the ratio of surface tensions of the droplets with/without surfactant.

Observation of red-tuning independent of the pump beam position suggests that in the surfactant-free case, WGM tuning is not dominated by droplet stretching which is associated with opposite tuning directions for off- 
axis and on-axis WGMs. Instead, as we discuss below, heating of the emulsion system by the optical stretching laser beams becomes significant, leading to temperature-induced red shifts observed in WGM spectral positions. Relatively large water absorption at the stretching laser wavelength of $1070 \mathrm{~nm}$ leads to an increase in the temperature of water and the droplet by more than $10^{\circ} \mathrm{C}$ per $1 \mathrm{~W}$ of $P_{\text {stretch }}{ }^{28}$ For viscous liquids such as immersion oil and water, the dominant mechanism of heat transfer on the scale of hundreds of microns is conduction. For the heat conductivity of water and immersion oil $\left(\sim 0.5 \mathrm{Wm}^{-1} \mathrm{~K}^{-1}\right)$, steady state is reached within $10-20 \mathrm{~ms}$ after temperature jumps of $10^{\circ} \mathrm{C}$. Since the duration of individual steps of the droplet tuning cycle is $>50 \mathrm{~ms}$, we can assume that the temperature distribution within the droplet reaches equilibrium before each spectral acquisition. Immediate results of such temperature changes are the droplet expansion and the change of the refractive index of the droplet and water. As mentioned in Section 5, interfacial tension does not change appreciably within the temperature range of $\sim 25^{\circ} \mathrm{C}$ above the room temperature. Therefore, temperature-induced changes of surface forces that could cause additional spectral tuning can be ruled out in the first approximation.

In order to predict the contributions of thermal expansion and refractive index changes to the WGM tuning slopes, we adopted an asymptotic formula which gives the spectral positions of WGMs in terms of oil/water relative refractive index $n_{1} / n_{2}$ and droplet radius $r_{0} .{ }^{29}$ This asymptotic formula holds for droplets with large size parameters $x=2 \pi r_{0} n_{2} / \lambda$ and its use is justified in our case where typical $r_{0} \approx 25 \mu \mathrm{m}$ and $\lambda \approx 625 \mathrm{~nm}$ give $x \approx 335$. First derivative of this asymptotic formula with respect to temperature gives the following expression for thermal tuning of WGM positions of spherical droplets:

$$
\begin{array}{r}
\frac{\Delta \lambda}{\Delta T}=\frac{\lambda \beta}{3}+\lambda \delta_{1}-\frac{\lambda^{2}}{2 \pi r n_{1}}\left(\frac{n_{2}}{n_{1}}\right)^{2}\left(\delta_{1}-\delta_{2}\right) \times \\
{\left[\left(1-\left(\frac{n_{2}}{n_{1}}\right)^{2}\right)^{-\frac{3}{2}}+2^{-\frac{1}{3}} \alpha_{1} \nu^{-\frac{2}{3}}\left(1-\left(\frac{n_{2}}{n_{1}}\right)^{2}\right)^{-\frac{5}{2}}\right]}
\end{array}
$$

where $\alpha_{1}=2.338$ is the first root of Airy function, $\nu=l+1 / 2$ with the angular mode number $l \approx 2 \pi r_{0} n_{1} / \lambda$, $\beta=3 \Delta r /(r \Delta T)=6.83 \times 10^{-4}{ }^{\circ} C^{-1}$ is the volume thermal expansion coefficient of immersion oil, and $\delta_{1}=$ $\Delta n_{1} /\left(n_{1} \Delta T\right)=-3.3 \times 10^{-4}{ }^{\circ} C^{-1}$ and $\delta_{2}=\Delta n_{2} /\left(n_{2} \Delta T\right)=-1.06 \times 10^{-4}{ }^{\circ} C^{-1}$ are the average thermal coefficients of the refractive index for immersion oil and water, respectively. For our system, $\frac{\Delta \lambda}{\Delta T}$ becomes $\sim 7 \mathrm{pm} /{ }^{\circ} \mathrm{C}$ which shows a good compensation of the thermal droplet expansion by the refractive index change. Assuming temperature increase of $\sim 10^{\circ} \mathrm{C} / \mathrm{W}$, the expected thermal tuning slope is approximately $0.07 \mathrm{~nm} / \mathrm{W}$. As thermally induced spectral tuning is isotropic within the droplet, tuning direction does not depend on the position of the pump beam focus on the droplet.

The above simple model of thermally induced tuning shows that in the off-axis excitation geometry, there are two competing mechanisms that tune the WGM spectral positions in opposite directions. While droplet stretching leads to a blue-tuning of WGMs, heating effects tune WGMs to the red end of the spectrum. The overall tuning slopes we observe in the experiments then result from the superposition of these two effects. For emulsions with surfactant, optical stretching dominates and the total wavelength tuning slope is negative (see Fig. 2a,b) whereas in the surfactant-free emulsions, the total wavelength tuning slope is positive due to the dominance of heating effects (see Fig. 3a,b). In the on-axis excitation geometry, both thermal and stretching tuning mechanisms lead to positive tuning slopes which explains why the measured tuning slope in Fig. 3c,d is more than the amount expected solely from the droplet deformation due to optical stretching.

In all experiments in which the excitation beam position was either on-axis or off-axis, we studied only WGMs propagating in a single plane of symmetry of an optically stretched droplet (polar or equatorial, respectively). Thus, it was not possible to monitor simultaneously the spectral positions of both blue-shifting and red-shifting WGMs for a single stretched droplet. However, such information is crucial for determining quantitatively the relative contribution of droplet stretching and thermal effects to the observed WGM tuning. In order to excite simultaneously WGMs propagating along different paths in a single droplet, the excitation beam position was moved to the droplet center (see Fig. 1b). Figure 4 summarizes experimental results obtained with on-center excitation of a $42 \mu \mathrm{m}$ diameter droplet for $\gamma=1.5 \mathrm{mN} / \mathrm{m}$. With on-axis excitation geometry, WGMs in the droplet fluorescence spectra display a spectral split $\delta \lambda$ that grows with increasing $P_{\text {stretch }}$ (see Fig. 4a). Within 
the single frequency-split WGM group, the shortest-wavelength WGM with $\lambda_{B}$ propagates in the equatorial plane whereas the longest-wavelength WGM with $\lambda_{R}$ propagates in the polar plane. As derived by Chen et al. ${ }^{11}$ wavelength $\lambda_{0}$ corresponding to the degenerate WGMs propagating in an undeformed spherical droplet is given by $\lambda_{0}=\lambda_{B}+2 \delta \lambda / 3$. This formula allows calculating the value of $\lambda_{0}$ for each stretching power from the experimental data. In case of pure stretching deformation of the droplet, $\lambda_{0}$ must be independent of $P_{\text {stretch }}$. Non-negligible thermal effects then induce changes of $\lambda_{0}$ with changing $P_{\text {stretch }}$. In Fig. $4 \mathrm{~b}$, values of $\lambda_{B}, \lambda_{R}$, and $\lambda_{0}$ are shown for WGM1, WGM2 labeled in Fig. 4a. Details of changes of $\lambda_{0}$ of the two WGMs with changing $P_{\text {stretch }}$ presented in Fig. 4c then clearly illustrate that the thermal tuning effects cannot be neglected. A linear fit of $\lambda_{0}$ as a function of $P_{\text {stretch }}$ given in Fig. $4 \mathrm{~d}$ yields the average thermal tuning slope of $0.085 \mathrm{~nm} / \mathrm{W}$. This value agrees well with our estimation of $0.077 \mathrm{~nm} / \mathrm{W}$ obtained from the above presented model of thermal tuning effects for the droplet studied in Fig. 4. Measured thermal tuning slope can be used to estimate pure stretching-induced tuning observed in the case of emulsions with surfactants (Fig. 2). For off-axis and on-axis excitations, the pure stretching tuning slopes given by the difference between total measured tuning slopes and the average thermal tuning slope become $-0.61 \mathrm{~nm} / \mathrm{W}$ and $0.41 \mathrm{~nm} / \mathrm{W}$, respectively. The experimental ratio of the stretching tuning slopes is then -1.5 which is close to the value of -2.0 predicted by the analytical formula for deformed droplet WGMs. The remaining difference between the experiments and prediction may be attributed to the nonuniform temperature distributions within droplets, and to the fact that individual measured tuning slopes were obtained with droplets of different diameters.
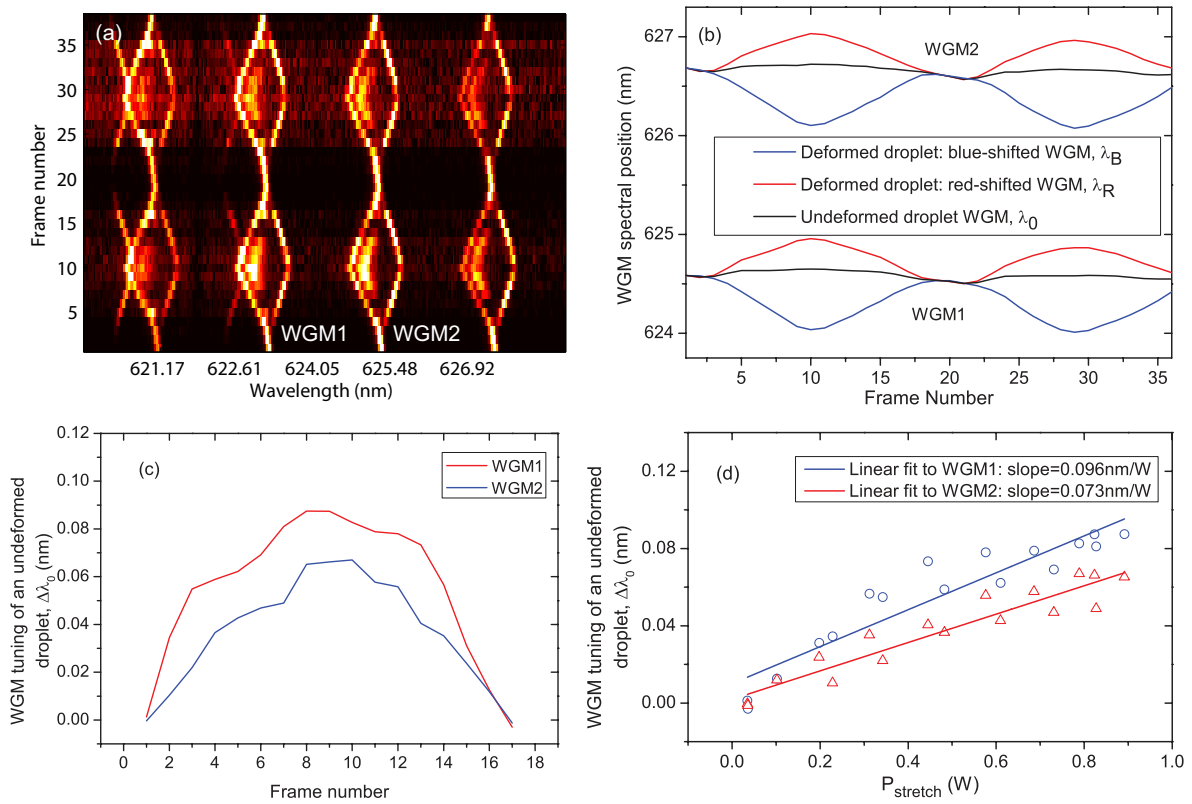

Figure 4. (a) Frequency-splitting of WGMs of a $42 \mu \mathrm{m}$ diameter droplet observed with on-center excitation geometry (see Fig. 1b). Light intensity in arbitrary units increases from black to white. (b) Dissolution-corrected spectral positions $\lambda_{B}, \lambda_{R}$, and $\lambda_{0}$ for WGM1, WGM2 labeled in (a) during two consecutive droplet tuning cycles. (c) Details of changes of spectral position $\lambda_{0}$ for WGM1, WGM2 labeled in (a) within a single droplet tuning cycle. (d) Changes of spectral position $\lambda_{0}$ for WGM1, WGM2 labeled in (a) as a function of the stretching laser power $P_{\text {stretch }}$ within a single droplet tuning cycle.

\section{CONCLUSION}

In this article, we have presented tunable optofluidic dye microlasers based on active optical resonant cavities formed by optically trapped microdroplets of fluorescently stained immersion oil emulsified in water. All-optical tuning of the laser emission wavelength was achieved by a controlled deformation of the droplet shape using light-induced forces generated by dual-beam optical trap. By lowering interfacial tension between the droplets and the aqueous host medium with a surfactant and sodium chloride, we have demonstrated almost reversible 
spectral tuning of the lasing droplet WGMs with tuning slopes up to $\sim 0.5 \mathrm{~nm}$ per Watt of the total stretching laser power. These spectral tuning slopes can be increased by further reduction of the droplet interfacial tension. Droplet liquid was observed to slightly dissolve in the host liquid during each tuning cycle, resulting in an irreversible blue-shift in the WGM spectral positions. This can be prevented by further optimization of the emulsion system in order to obtain fully reversible spectral tuning of the WGMs. Tuning direction of our liquid-cavity microlasers could be easily reversed by changing the position of the optical pumping beam on the droplet. Control experiments with surfactant-free emulsion droplets revealed an additional mechanism of reversible spectral tuning triggered by thermally induced changes of the droplet size and refractive index of both the droplet and the host medium. Such thermally induced tuning is a consequence of the absorption of the near-infrared optical stretcher light in water; it can be avoided by changing the wavelength of the used laser beam or replacing aqueous host medium with deuterium oxide that has minimal absorption in the near-infrared spectral region. Our droplet-based tunable and disposable optofluidic lasers can be used with a wide choice of fluorescent dyes and they can be easily integrated into microfluidic lab-on-a-chip platforms. Thus, they represent an attractive alternative for tunable sources of light for on-chip applications in chemical and biological analysis and sensing.

\section{ACKNOWLEDGMENTS}

The authors acknowledge support from TÜBİTAK (Grant No. 111T059), Czech Science Foundation (P205/11/P294), Technology Agency of the Czech Republic (TA03010642), Ministry of Education, Youth and Sports of the Czech Republic together with the European Commission (ALISI CZ.1.05/2.1.00/01.0017). The authors thank Martin Šiler for help with thermal analysis and A. L. Demirel for help with the surface tension measurements.

\section{REFERENCES}

[1] Datsyuk, V. V., "Optics of microdroplets," J. Mol. Liq. 84, 1308-1316 (2001).

[2] Righini, G. C., Dumeige, Y., Feron, P., Ferrari, M., Conti, G. N., Ristic, D., and Soria, S., "Whispering gallery mode microresonators: Fundamentals and applications," Rivista Del Nuovo Cimento 34, 435-488 (2011).

[3] Vahala, K. J., "Optical microcavities," Nature 424, 839 (2003).

[4] Psaltis, D., Quake, S. R., and Yang, C., "Developing optofluidic technology through the fusion of microfluidics and optics," Nature 442, 381-386 (2006).

[5] Qian, S.-X., Snow, J. B., Tzeng, H. M., and Chang, R. K., "Lasing droplets: Highlighting the liquid-air interface by laser emission," Science 231, 486 (1986).

[6] Snow, J. B., Qian, S.-X., and Chang, R. K., "Stimulated Raman scattering from individual water and ethanol droplets at morphology-dependent resonances," Opt. Lett. 10, 37-39 (1985).

[7] Sennaroglu, A., Kiraz, A., Dündar, M. A., Kurt, A., and Demirel, A. L., "Raman lasing near $630 \mathrm{~nm}$ from stationary glycerol-water microdroplets on a superhydrophobic surface," Opt. Lett. 32, 2197-2199 (2007).

[8] Kiraz, A., Kurt, A., Dündar, M. A., and Demirel, A. L., "Simple largely tunable optical microcavity," Appl. Phys. Lett. 89, 081118 (2006).

[9] Kiraz, A., Karadağ, Y., and Coskun, A. F., "Spectral tuning of liquid microdroplets standing on a superhydrophobic surface using electrowetting," Appl. Phys. Lett. 92, 191104 (2008).

[10] Kiraz, A., Karadag, Y., Yorulmaz, S. C., and Muradoglu, M., "Reversible photothermal tuning of a salty water microdroplet," Phys. Chem. Chem. Phys. 11, 2597-2560 (2009).

[11] Chen, G., Mazumder, M. M., Chemla, Y. R., Serpengüzel, A., Chang, R. K., and Hill, S. C., "Wavelength variation of laser emission along the entire rim of slightly deformed microdroplets," Opt. Lett. 18, 1993-1995 (1993).

[12] Yorulmaz, S. C., Mestre, M., Muradoglu, M., Alaca, B. E., and Kiraz, A., "Controlled observation of nondegenerate cavity modes in a microdroplet on a superhydrophobic surface," Opt. Commun. 282, 30243027 (2009).

[13] Saito, M., Shimatani, H., and Naruhashi, H., "Tunable whispering gallery mode emission from a microdroplet in elastomer," Optics Express 16, 11915-11919 (2008). 
[14] Humar, M., Ravnik, M., Pajk, S., and Muševič, I., "Electrically tunable liquid crystal optical microresonators," Nature Photonics 3, 595-600 (2009).

[15] Tanyeri, M., Perron, R., and Kennedy, I. M., "Lasing droplets in a microfabricated channel," Optics Letters 32, 2529-2531 (2007).

[16] Aas, M., Jonáš, A., and Kiraz, A., "Lasing in optically manipulated, dye-doped emulsion microdroplets," Opt. Commun. 290, 183-187 (2013).

[17] Tang, S. K. Y., Li, Z., Abate, A. R., Agresti, J. J., Weitz, D. A., Psaltisd, D., and Whitesides, G. M., "A multi-color fast-switching microfluidic droplet dye laser," Lab on a Chip 9, 2767-2771 (2009).

[18] Tang, S. K. Y., Derda, R., Quan, Q., Loncar, M., and Whitesides, G. M., "Continuously tunable microdroplet-laser in a microfluidic channel," Opt. Express 19, 2204-2215 (2011).

[19] Guck, J., Ananthakrishnan, R., Mahmood, H., Moon, T. J., Cunningham, C. C., and Käs, J., "The optical stretcher: A novel laser tool to micromanipulate cells," Biophys. J. 81, 767-784 (2001).

[20] Ashkin, A., "Acceleration and trapping of particles by radiation pressure," Physical Review Letters 24, 156-159 (1970).

[21] Constable, A., Kim, J., Mervis, J., Zarinetchi, F., and Prentiss, M., "Demonstration of a-fiber-optical light-force trap," Optics Letters 18(21), 1867-1869 (1993).

[22] Čižmár, T., Brzobohatý, O., Dholakia, K., and Zemánek, P., "The holographic optical micro-manipulation system based on counter-propagating beams," Laser Physics Letters 8, 50-56 (2011).

[23] Møller, P. C. F. and Oddershede, L. B., "Quantification of droplet deformation by electromagnetic trapping," Europhys. Lett. 88, 48005 (2009).

[24] Ward, A. D., Berry, M. G., Mellorb, C. D., and Bain, C. D., "Optical sculpture: controlled deformation of emulsion droplets with ultralow interfacial tensions using optical tweezers," Chemical Communications 43, 4515-4517 (2006).

[25] Holler, S., Goddard, N. L., and Arnold, S., "Spontaneous emission spectra from microdroplets," Journal of Chemical Physics 108, 6545 (1998).

[26] Drelich, J., Fang, C., and White, C. L., [The Encyclopedia of Surface and Colloid Science: Measurement of Interfacial Tension in Fluid/Fluid Systems], Marcel- Dekker (2002).

[27] Aveyard, R., Binks, B., Clark, S., and Mead, J., "Interfacial tension minima in oil-water-durfactant systrms," J. Chem. Soc., Faraday Trans. 82, 125-142 (1986).

[28] Ebert, S., Travis, K., Lincoln, B., and Guck, J., "Fluorescence ratio thermometry in a microfluidic dualbeam laser trap," Optics Express 15, 15493-15499 (2007).

[29] Lam, C. C., Leung, P. T., and Young, K., "Explicit asymptotic formulas for the positions, widths, and strengths of resonances in Mie scattering," J. Opt. Soc. Am. B 9, 1585-1592 (1992). 